Pesq. Vet. Bras. 36(4):329-338, abril 2016

DOI: 10.1590/S0100-736X2016000400013

\title{
Estudo morfométrico de rins em felinos domésticos (Felis catus ${ }^{1}$
}

\author{
Rafael G. Agopian²*, Katia P. Guimarães², Renata A. Fernandes², Marcos Vinícius \\ M. Silva ${ }^{2}$, Marta M.S. Righetti ${ }^{3}$, Cleide Rosana Duarte Prisco ${ }^{3}$, Pedro P. Bombonato ${ }^{2}$ \\ e Edson Aparecido Liberti ${ }^{3}$
}

\begin{abstract}
Agopian R.G., Guimarães K.P., Fernandes R.A., Silva M.V.M., Righetti M.M.S., Prisco C.R.D., Bombonato P.P. \& Liberti E.A. 2016. [Morphometric study on the kidneys of domestic cats (Felis catus).] Estudo morfométrico em rins de felinos domésticos (Felis catus). Pesquisa Veterinária Brasileira 36(4):329-338. Departamento de Cirurgia, Faculdade de Medicina Veterinária e Zootecnia, Universidade de São Paulo, Av. Prof. Dr. Orlando Marques de Paiva 87, São Paulo, SP 05508-270, Brazil. E-mail: rafael.agopian@gmail.com

Renal health has over the last few years called attention of veterinarians, because the chronic failure of the kidneyu is a major cause of morbidity and mortality in cats. This paper proposes biometric characterization of the kidneys from cats, Felis catus, on the macroscopic aspects (length, height, width, weight and volume), mesoscopic (height of the cortex and medulla, and their inter-relationship) and microscopic (glomerular volume) in order to establish possible differences arising from age, gender and bilateral symmetry. It where used 30 kidneys of animals from specie Felis catus (cat), 15 males and 15 females with ages between 3 months to 15 years, divided into three groups: group 1 (3-9 months), group 2 ( 3 - 5 years) and group 3 (above 10 years). The kidneys were photographed, weighed, measured and processed for histology. The glomerular volume was obtained by stereology. The macroscopic data allowed to observe a morphometric and morphologic impartiality when compared sides, regardless of group and sex, about the length, height, width, weight and kidney volume. Significant difference was detected between males and females regardless of age for length, height, weight and volume, except for the width. There was a significant difference for all parameters considering the age: length, height, width, weight and volume. Wasnt detected signficant diferences between left and right kdney measurements of cortex, medulla and their inter-relationship, when considered bilateral symmetry. No difference was detected between males and females in mesoscopy in the cortex, medulla and cortex interrelationship cortex/medulla. Regarding age, the measurement of the height of the cortex and the interrelationship cortex/medulla showed a significant difference, but this difference was not established to the medulla of the kidney. Significant differences were found between groups, males and females, compared to the volume occupied by the glomerulus on the parenchyma renal. In all of biometric techniques there wasnt differences between right and left kidneys. The macroscopic measurements showed that males had larger kidney when compared length, height, width, weight and volume than females. The age range is directly related to the size of the kidney, which grows in all dimensions, gets heavier and with higher volume when compared young adults, and shows morphometry stability in relation to adults and senile. The renal parenchyma did not differ between the sexes, but the height of the cortex and the medulla ratio increases with age. The volume of the renal glomerulus is higher in males than in females and also increases in with the age. The kidneys showed a predominance of type I collagen in group 1 and 2, and type III collagen in group 3.
\end{abstract}

INDEX TERMS: Felis catus, cats, stereology, kidney, collagen.

\footnotetext{
${ }^{1}$ Recebido em 25 de junho de 2015.

Aceito para publicação em 17 de janeiro de 2016.

${ }^{2}$ Departamento de Cirurgia, Faculdade de Medicina Veterinária e Zootecnia (FMVZ), Universidade de São Paulo (USP). Rua Prof. Dr. Orlando
}

\footnotetext{
Marques de Paiva 87. Cidade Universitária, São Paulo, SP 05508-270, Brasil.*Autor para correspondência: rafael.agopian@gmail.com

${ }^{3}$ Departamento de Anatomia, Instituto de Ciências Biomédicas (ICB), USP, Av. Prof. Dr. Lineu Prestes 2415, São Paulo, SP 05508-000, Brasil.
} 
RESUMO.- A saúde renal tem ao longo dos últimos anos chamado atenção dos médicos veterinários, pois o comprometimento deste órgão na insuficiência renal crônica se apresenta como a maior causa de morbidade e mortalidade em felinos. 0 presente estudo propõe a caracterização biométrica dos rins de gatos, Felis catus, sob os aspectos macroscópicos (comprimento, altura, largura, peso e volume), mesoscópico (altura do córtex e da medula, e a sua inter-relação) e microscópica (volume glomerular), a fim de se estabelecer possíveis diferenças decorrentes de idade, sexo e simetria bilateral. Foram utilizados, rins de 30 animais da espécie Felis catus (gato deméstico), sendo 15 machos e 15 fêmeas com idade variando entre 3 meses a 15 anos, divididos em três grupos: grupo 1 (3-9 meses), grupo 2 (3-5 anos) e grupo 3 (acima de 10 anos). Os rins foram fotografados, pesados, mensurados e processados para histologia. 0 volume glomerular foi obtido através de estereologia. Os dados macroscópicos permitiram observar uma imparcialidade morfométrica e morfológica quando comparados os lados, independente do grupo e do sexo, sobre o comprimento, altura, largura, peso e volume dos rins. Para o sexo foi detectado diferença significante entre machos e fêmeas independente da faixa etária para comprimento, altura, peso e volume, com exceção da largura. Considerando a idade houve diferença significativa para todos os parâmetros: comprimento, altura, largura, peso e volume. Nas mensurações de córtex, medula e sua inter-relação, quando considerada a simetria bilateral, não foi detectada diferença significante entre rim direito e esquerdo. Para o sexo, não foi detectada diferença entre machos e fêmeas na mesoscopia no córtex, na medula e na inter-relação córtex/ medula. Quanto à idade, a mensuração da altura do córtex e a inter-relação córtex/medula apresentou diferença significante, porém essa diferença não foi estabelecida para a medula do rim. Foram encontradas diferenças significantes entre grupos e entre machos e fêmeas, em relação ao volume ocupado pelo glomérulo no parênquima renal. Em todas as técnicas biométricas utilizadas não foram detectadas diferenças entre os rins direito e esquerdo. As mensurações macroscópicas mostraram que machos apresentaram um rim maior em comprimento, altura, largura, peso e volume do que as fêmeas. A faixa etária está diretamente relacionada ao tamanho do rim, que cresce em todas as dimensões, fica mais pesado e com maior volume quando comparados jovens e adultos, e se mostra estável morfometricamente em relação aos adultos e senis. 0 parênquima renal não difere entre os sexos, mas a altura do córtex e a relação com a medula aumentam com a idade. 0 volume do glomérulo renal é maior em machos do que em fêmeas e também aumenta em relação à idade. Os rins mostraram um predomínio de colágeno tipo I nos animais do grupo 1 e 2, e colágeno tipo III nos animais do grupo 3.

TERMOS DE INDEXAÇÃO: Felis catus, felinos, estereologia, rim, colágeno.

\section{INTRODUÇÃO}

Diante da relevância dos rins em processos fisiológicos, bem como a manutenção da homeostasia do organismo, participando desde o equilíbrio hídrico e eletrolítico até atuação endócrina com a produção de renina e eritropoietina. Estudos para a determinação de parâmetros biométricos renais são fundamentais, já que estes podem servir de referência para futuras avaliações clínicas.

As informações obtidas em estudos morfométricos determinam parâmetros que podem ser usados como referência tanto em estudos clínicos quanto para comparar possíveis alterações decorrentes de diversas enfermidades. Alguns deles podem ressaltar a importância da compreensão do funcionamento dos rins.

Em cães de grande porte, por exemplo, de 1.000 a 2.000 litros de sangue perfundem diariamente os rins, obtendo-se 200 a 300 litros de líquido filtrado que são, posteriormente, reduzidos por reabsorção até que 1 ou 2 litros de urina remanescentes sejam eliminados (Dyce et al. 2004).

As enfermidades renais em cães e principalmente nos felinos tem sido abordada em pesquisas veterinárias, dentre as quais pode-se destacar a insuficiência renal, por ser uma condição patológica relacionada à perda funcional do rim, que pode ser parcial, completa ou irreversível (Crepaldi et al. 2008).

As doenças renais são causadas por problemas de diversas ordens etiológicas e induzem alterações estruturais e funcionais, diagnosticadas através de biópsia para histopatologia (Spargos \& Haas 1994).

Estudos histológicos contribuem para a classificação e identificação de doença glomerular, cuja classificação de acordo com o padrão histológico das alterações do parênquima renal, fornece subsídios necessários para a compreensão da doença (Stevens \& Lowe 1998).

Pesquisas que avaliam aspectos macroscópicos, topográficos e microscópicos de diferentes órgãos e sistemas contribuem para a obtenção de informações sobre a morfologia normal do corpo, bem como para a compreensão das diferentes patologias que acometem tais estruturas, o que permite avançar em todas as áreas da ciência médica.

Os parâmetros morfológicos e morfométricos são utilizados na rotina clínica como referência de sanidade do rim, em especial nos exames de ultrassonografia renal, onde a avaliação de tamanho, volume e aspectos do parênquima, tais como ecogenicidade do córtex e da medula renal, e as definições imagológicas destas camadas, são dados que permitem estabelecer a higidez ou não do órgão (Kealy \& Mcallister 2005).

Em experimentos que se utilizam de extratos herbais, fármacos ou promoção de doenças, como diabetes, a avaliação morfométrica dos glomérulos é utilizada em que pesquisas envolvem animais e humanos, como forma de se estabelecer parâmetros morfométricos comparativos entre etnias, sexo, idade ou mesmo como referência da presença de lesão renal com ou sem alterações fisiológicas (McNamara et al. 2009, Agopian 2010, Almeida et al. 2013).

Considerando que para a espécie felina, a doença renal aparece como uma das principais causas de morbidade e mortalidade (Crepaldi et al. 2008), estudos que viabilizem informações sobre os aspectos relacionados aos rins nessa espécie são relevantes. Neste contexto, a proposta do presente trabalho foi a de desenvolver uma análise macroscópica, microscópica e morfométrica dos rins desta espécie 
com o intuito de estabelecer possíveis alterações relacionadas, não aos aspectos patológicos, mas às condições decorrentes do envelhecimento normal.

\section{MATERIAL E MÉTODOS}

Os rins foram obtidos de 30 animais da espécie Felis catus (gato doméstico), sendo 15 machos e 15 fêmeas com idade variando entre 3 meses a 15 anos, oriundos de clínicas, hospitais veterinários e campanhas de esterilização cirúrgica, que vieram à óbito em decorrência de causas naturais. 0 trabalho foi conduzido após aprovação do Comitê de Ética para o Uso de Animais da FMVZ-USP, Protocolo no 3035/2013.

De acordo com a idade, os espécimes foram divididos nos grupos 1 , 2 e 3 ( $n=10 ; 5$ machos e 5 fêmeas por grupo), sendo o grupo 1 (G1) composto por animais com idade entre 3 e 9 meses; o grupo 2 (G2), por animais com idade entre 3 e 5 anos e o grupo 3 (G3), por animais com idade acima de 10 anos.

A análise macroscópica foi realizada após a retirada dos rins direito e esquerdo da cavidade abdominal, dos animais de todos os grupos, foram inicialmente determinadas as mensurações macroscópicas (comprimento, altura e largura), com o auxílio de paquímetro digital (Mitutoyo ${ }^{\circledR}$ modelo CD-8" CX-B).

Para o comprimento renal foram tomados como referência os pontos mais extremos dos polos cranial e caudal. A mensuração da altura baseou-se na distância entre o hilo renal e o ponto médio na margem lateral do rim, entre os polos cranial e caudal. A largura foi determinada entre os pontos médios das faces dorsal e ventral, estabelecidos no centro da medida da altura em cada face.

0 peso em gramas foi obtido através de balança digital semi-analítica SHIMADZU ${ }^{\circledR}$ modelo BL $3200 \mathrm{H}$.

0 volume foi determinado pela técnica de volumetria por deslocamento de líquido, conforme preconizado por Mandarim de Lacerda (1994).

Após a obtenção dos dados macroscópicos os rins foram seccionados por um corte no plano frontal, obtendo-se assim duas metades paquiméricas para avaliação da arquitetura interna e preparo para as etapas meso e microscópicas.

Para processamento histológico, foi utilizada uma metade de cada rim de cada espécime de todos os grupos obtida através de secção sagital, onde se distinguiu as camadas cortical e medular do parênquima. Ao mesmos foram fixados em solução de formol (10\%), desidratados em série crescente de alcoóis (50\% ao absoluto), diafanizados em xilol e incluídos em parafina, e então os espécimes foram submetidos a cortes semi-seriados de 6 $\mu \mathrm{m}$ de espessura, foram utilizados 10 cortes de cada paquímero renal escolhido para cada espécime e corados pelos métodos da Hematoxilina-Eosina (HE) (Tolosa 2003) e do Picrosirius (Junqueira et al. 1979). A avaliação histológica foi realizada utilizando-se um microscópico binocular (Axioscope; Zeiss ${ }^{\circledR}$ ) acoplado a um sistema de processamento de imagem computadorizada (Zeiss, Axiovision Rel. 4.6).

Os cortes corados em Picrosirius foram avaliados através do auxílio de lentes para polarização das imagens, capturadas e registradas em fotomicrografias para a descrição do padrão de deposição dos tipos I e III de fibras colágenas. Dos 14 cortes em HE representando rins direito e esquerdo de cada animal, foram selecionados, aleatoriamente, 7 (contemplando rim direito e esquerdo) para a análise estereológica.

Para a Determinação da Densidade de Volume Glomerular, foram adotados princípios estereológicos, onde a quantificação bidimensional pode ser utilizada como ferramenta eficiente para a obtenção de estimativas quantitativas em estruturas tridimensionais optou-se pela utilização do método Nucleator, que consegue estabelecer o volume ocupado por uma estrutura circular em um tecido através de medidas de comprimento de raio desta, a partir de um ponto fixo (Gundersen et al. 1988).

Para a aplicação do método, utilizou-se 7 lâminas contendo um corte histológico dos rins de cada espécime, onde foram fotografados 2 campos para cada corte, com objetiva de $20 \mathrm{x}$, para a visualização dos corpúsculos renais distribuídos pelo córtex. Nestes campos, selecionou-se um total de 5 glomérulos por corte $(2$ e 3 por campo). A técnica para a aplicação do Nucleator foi estabelecida a partir das mensurações desses 5 glomérulos, totalizando 350 glomérulos por grupo.

A mensuração dos glomérulos com a régua gerou a marcação de dois domínios, os quais foram registrados em uma tabela modelo com variáveis definidas para aplicação do método Nucleator.

Com os registros dos domínios na coluna G da tabela foi possível calcular os índices da coluna $\mathrm{H}$ e obter o volume $\left(\mu \mathrm{m}^{3}\right)$ ocupado pelos glomérulos no parênquima renal através das fórmulas:

$$
\overline{l_{\mathrm{n}}^{3}}=\sum_{\sum G}^{\sum H}=\frac{-\mu \mathrm{m}}{V_{N}}=\overline{4 \pi} \cdot l_{\mathrm{n}}^{3}=\frac{\mu \mathrm{m}}{\mathrm{n}}
$$

Para a mesoscopia, os cortes corados com HE tiveram a determinação das alturas representadas pelo córtex e medula renais, realizadas através da aquisição de imagens com o auxílio de uma lupa estereoscópica (Carl Zeiss Microimaging, modelo Stemi® SV6), acoplada a uma câmera fotográfica digital (Power shot A640, Canon). 0 processamento das imagens foi realizado em equipamento de imagem computadorizada (Zeiss, Axiovision Rel. 4.6). Nesta etapa foi utilizado aumento de 0,8 vezes e determinadas as medidas do córtex e da medula $(\mu \mathrm{m})$ através de sistema de medidas em barras calibradas do programa Axiovision.

A análise estatística foi apresentada com média \pm desvio padrão com os dados relativos a comprimento, altura, largura, peso e volume renais, morfometria do córtex, da medula e da inter-relação córtex / medula, bem como a densidade de volume glomerular. A densidade de volume glomerular foi ainda analisada empregando-se análise de variância - ANOVA com dois fatores: grupo e sexo, seguida de comparações múltiplas pelo método de Tukey.

Para as demais variáveis de interesse, foi empregada a análise de variância com medidas repetidas - ANOVA com três fatores: grupo, sexo e lado (medida obtida no mesmo animal), seguida por comparações múltiplas pelo método de Bonferroni.

Foram consideradas diferenças significativas valores de $\mathrm{p}<$ 0,05 .

\section{RESULTADOS}

Todos os espécimes apresentaram os rins inteiramente formados sem nenhum tipo de alteração em sua topografia. Todos os animais estavam em perfeito estado de conservação e não apresentavam nenhum tipo de má formação congênita.

Com a utilização deste protocolo experimental foi obtido uma divisão de grupos homogêneos, sempre estabelecendo uma proporção igualitária de machos e fêmeas para cada um dos grupos.

Para a morfometria macroscópica os dados de comprimento, altura, largura, peso e volume dos rins foram analisados levando em consideração o lado (simetria bilateral), o sexo e a idade (Fig.1).

Quando comparados os lados não foi detectada diferença significante entre os rins direito e esquerdo, independente do grupo e do sexo, referente aos parâmetros comprimento $(\mathrm{p}=0,460)$, altura $(\mathrm{p}=0,352)$, largura $(\mathrm{p}=0,068)$, peso $(p=0,321)$ e volume $(p=0,076)$. 

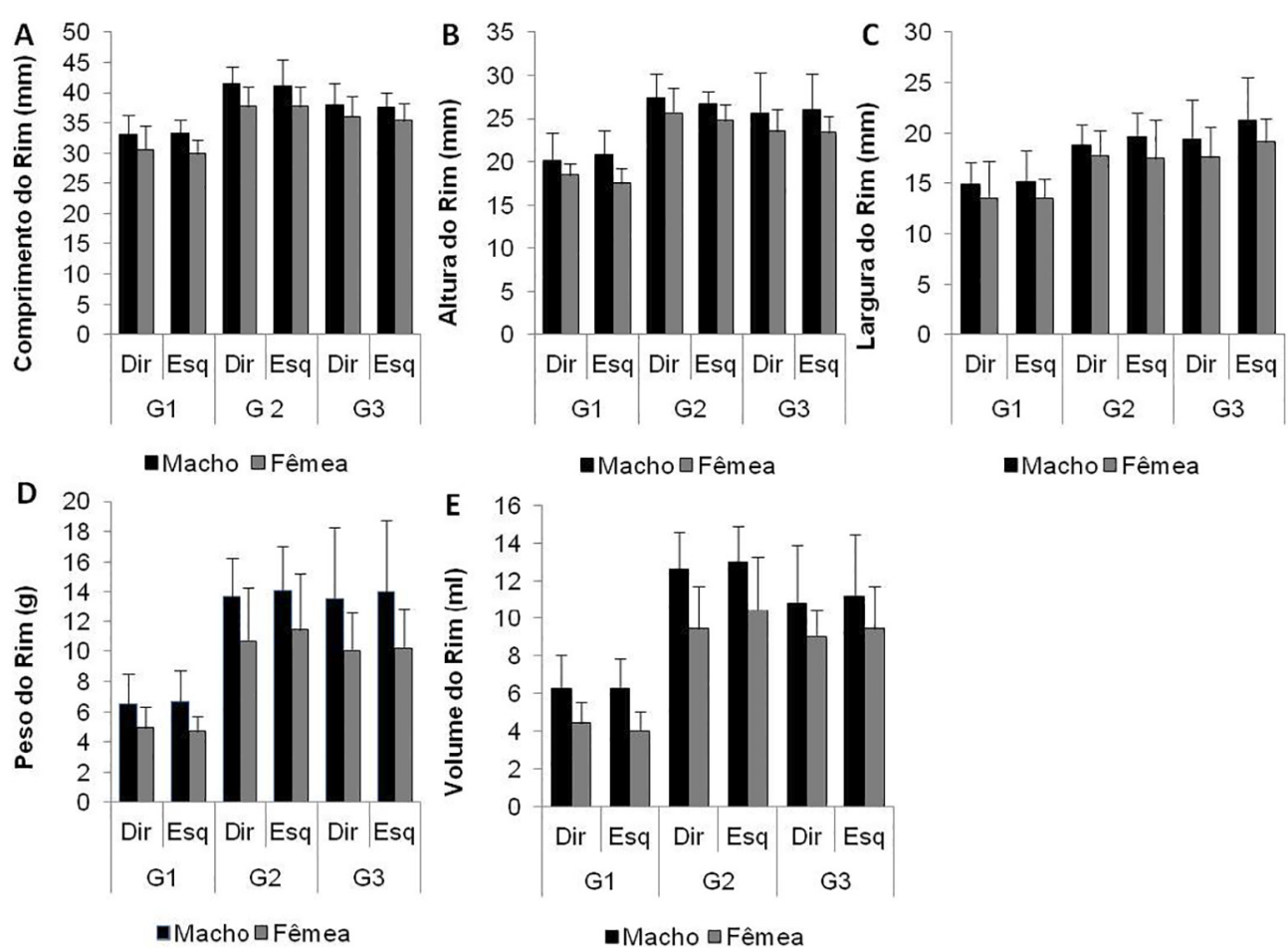

Fig.1. Média e Desvio Padrão dos rins direito e esquerdo dos machos e fêmeas de Felis catus dos grupos 1, 2 e 3. (A) Comprimento (mm). (B) Altura (mm). (C) Largura (mm). (D) Peso (g). (E) Volume (ml).

Em relação ao sexo dos animais, a análise detectou diferença significante entre machos e fêmeas, independente da faixa etária para as variáveis comprimento $(\mathrm{p}=0,015)$, altura $(\mathrm{p}=0,036)$, peso $(\mathrm{p}=0,014)$ e volume $(\mathrm{p}=0,008)$, com exceção da largura $(\mathrm{p}=0,121)$, que não apresentou diferença significativa.

Nesta etapa analítica evidenciou-se a diferença significativa referente ao tamanho dos rins onde, independente do grupo, os machos apresentaram maior tamanho renal em relação às fêmeas.

Considerando-se a idade (grupo 1, jovem; grupo 2, adulto; e grupo 3 , senil), houve diferença significativa para todos os parâmetros morfométricos: comprimento $(\mathrm{p}<0,001)$, altura $(p<0,001)$, largura $(p=0,001)$, peso $(p<0,001)$ e volume $(\mathrm{p}<0,001)$.

Os indivíduos do G1 apresentam rins menores que os G2 e G3 ( $p<0,05)$, porém, não houve diferença significante quando G2 e G3 foram comparados ( $p>0,05$ ).

Os dados demonstram um crescimento do rim proporcional à idade, ou seja, há uma evolução ascendente de jovem para adulto, e de jovem para senil. 0 fato de não haver diferença significante entre G2 e G3 indica uma estabilidade morfométrica do órgão nessas fases da vida.

Para a mesoscopia foram obtidos dados das mensurações do córtex, da medula e sua inter-relação (Fig.2). Quando considerada a simetria bilateral, não foi detectada diferença significante entre o rim direito e o rim esquerdo ( $p=0,205, p=0,438$ e $p=0,589$, respectivamente).

Em relação ao fator sexo, não foi detectada diferença entre machos e fêmeas para todas as mensurações mesoscópicas: córtex $(\mathrm{p}=0,093)$, medula $(\mathrm{p}=0,245)$ e inter-relação córtex / medula $(p=0,414)$. Quando considerado o fator idade, a mensuração da altura do córtex e a inter-relação córtex / medula apresentou diferença significante $(p=0,001$ e $p=0,028$, respectivamente), porém, essa diferença não foi estabelecida na mensuração da altura da camada medular do $\operatorname{rim}(\mathrm{p}=0,078)$.

Tais resultados demonstram que as mensurações do córtex apresentaram diferença significante $(p<0,05)$ quando comparados os grupos G1 e G2, e G1 e G3. Também para esse parâmetro, não foi detectada diferença significante entre os grupos G2 e G3 (p>0,05). Entretanto, a inter-relação córtex / medula apresentou diferença significante apenas entre jovens e adultos $(\mathrm{p}<0,05)$.

Para a análise microscópica, foram realizadas avaliações quantitativa e qualitativa (Fig.3), das lâminas coradas em Picrosirius. A avaliação quantitativa determinou o volume glomerular no parênquima renal e foi baseada em princípios estereológicos, onde foi adotada a técnica do Nucleator. Para a análise estatística foram considerados apenas os fatores idade e sexo.

A Análise de Variância empregada detectou diferenças significantes entre grupos $(\mathrm{p}<0,001)$ independente do sexo e entre machos e fêmeas independente do grupo $(p<0,001)$.

Para o volume ocupado pelo glomérulo no parênquima renal os dados obtidos pelo método estereológico empregado mostram que existe diferença deste volume quando comparamos animais de idades diferentes. Os jovens apresentam menor volume glomerular quando comparados aos adultos e senis e os adultos apresentam menor volume glomerular quando comparados aos senis, $\mathrm{p}<0,05$, ou seja, houve um aumento no volume glomerular médio em relação à idade.

Existe também um comportamento diferente entre os 

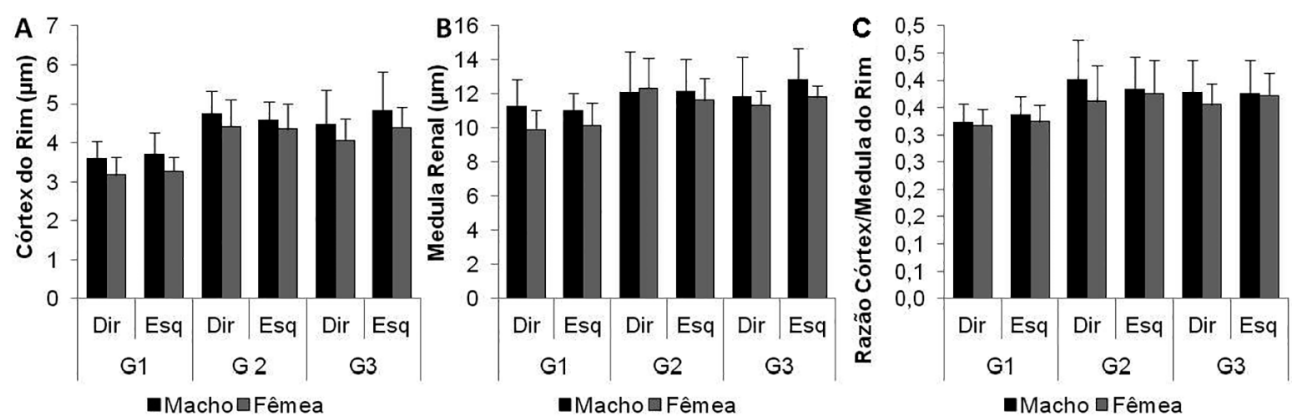

Fig.2. Média e Desvio Padrão dos rins direito e esquerdo de Felis catus machos e fêmeas dos grupos 1, 2 e 3. (A) Altura do córtex $(\mu \mathrm{m})$. (B) Altura da medula $(\mu \mathrm{m})$. (C) Razão córtex/medula.

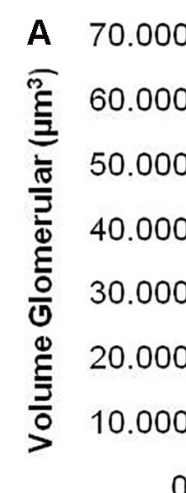

0

G1

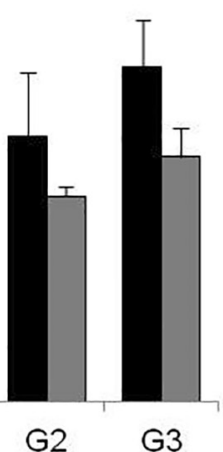

G3

-Macho rFêmea
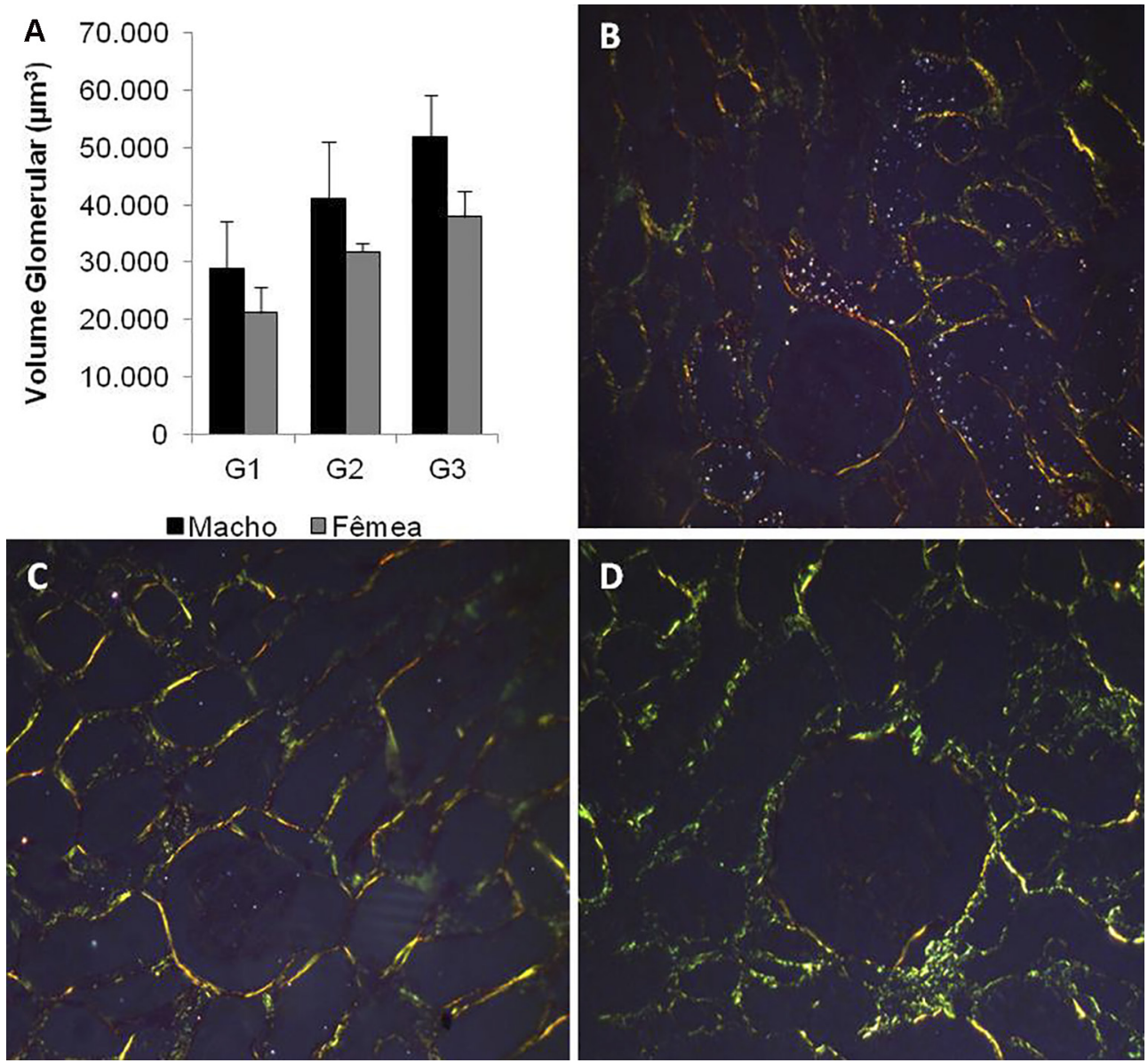

Fig.3. (A) Média e Desvio Padrão do volume glomerular ( $\mu \mathrm{m} 3$ ) dos rins de Felis catus machos e fêmeas dos grupos 1, 2 e 3. Aspectos da deposição de colágenos: (B) em animais do grupo 1, (C) em animais do grupo 2, (D) em animais do grupo 3.

animais quando considerado o sexo, a análise estatística mostrou diferença significante entre machos e fêmeas, indicando que o volume glomerular é maior no macho, ou seja, quando levado em consideração o fator sexo, independente da idade, machos possuem maior volume glomerular no parênquima renal que fêmeas, $\mathrm{p}<0,001$.

A avaliação qualitativa dos cortes histológicos foi realizada através das imagens obtidas de lâminas coradas em Picrosirius, permitindo verificar a distribuição da deposição de colágeno no córtex renal, onde se localizam os glomérulos. Na visualização dos cortes histológicos corados em Picrosirius, os animais avaliados dos grupos 1 e 2 apre- sentaram um predomínio de deposição de colágeno tipo I (coloração amarela e vermelha), já para o grupo 3, houve um predomínio de deposição de colágeno tipo III (verde), nas avaliações de fotomicrografias em luz polarizada do córtex renal destes animais.

\section{DISCUSSÃO}

As doenças que comprometem morfologia e função dos rins em felinos, já são descritas como a segunda maior causa de mortes nesses animais e têm no seu diagnóstico preciso e precoce a melhor ferramenta para uma terapia eficiente e resolutiva (Crepaldi et al. 2008, Schenck \& Chew 2010). 
As alterações morfológicas e funcionais a cerca das doenças renais podem acometer os glomérulos, os túbulos, o tecido intersticial e ou os vasos sanguíneos. As afecções renais podem ser de origem hereditária e ou congênita, infecciosa e tóxica (toxinas endógena ou exógena), imunomediada, por desequilíbrios eletrolíticos (hipercalcemia e hipocalemia no felino) e traumática (Polzin 2011).

Estudos sobre a incidência de doenças renais mostram que 1,6 a $20 \%$ da população de gatos desenvolvem a doença de forma crônica. Mesmo podendo acometer gatos de todas as idades, o envelhecimento é um fator importante no aparecimento desta doença, torna-se mais frequente nos animais idosos, com idade superior a 7 anos. Estima se que até $49 \%$ dos gatos com idade superior a 15 anos são acometidos. Tem sido relatada que, quando comparada com cães, a incidência de DRC espontânea em felinos senis é cerca de três vezes mais alta (Ross et al. 2006, Amador 2009, Polzin 2011).

A disposição das idades neste experimento levou em consideração estes relatos, onde a avaliação morfométrica e morfológica foi estabelecida a partir de 3 grupos distintos, com idades variadas, o que promoveu a avaliação destes parâmetros entre animais jovens (3-9 meses), adultos (3-5 anos) e senis (acima de 10 anos).

Diferenças significativas entre as idades foram notadas em todos os parâmetros morfométricos: macroscópicos (comprimento, altura, largura, peso e volume dos rins), mesoscópicos (altura do córtex e inter-relação córtex / medula e microscópicos (densidade de volume glomerular). Além destes, destacou-se a avaliação na estrutura de colágeno do grupo 3, comparativamente aos grupos 1 e 2 .

A observação destes resultados confirma a influência da idade na diferenciação morfológica do órgão, como descrito por diferentes autores, e serve de alerta na prevenção do aparecimento de comprometimento dos rins com o avançar da idade em felinos. Como descrito por Slusarz et al. (2013), tal confirmação serve também para humanos, uma vez que a incidência de doenças renais em indivíduos norte americanos, aumentou, proporcionalmente ao aumento da população acima de 65 anos.

Na avaliação morfométrica macroscópica aplicada neste estudo, além da idade, foi considerado como fator para comparação o sexo e a simetria bilateral. Estudos de populações sobre o acometimento dos animais por afecções dos rins levam em consideração tais fatores para comparar possíveis diferenças entre eles, entretanto, os achados divergem sobre a relação direta destes fatores nas alterações renais.

Alguns autores não verificaram diferenças entre felinos machos e fêmeas quanto ao acometimento de doenças renais. É o caso do estudo de DiBartola et al. (1987), que ao avaliarem 74 gatos acometidos por doença renal, onde a maioria dos animais tinha idades superiores a 7 anos, não detectaram nenhuma predileção por raça ou sexo, da mesma forma, Elliott \& Barber (1998), relataram os achados clínicos em 80 gatos diagnosticados com insuficiência renal em diversos estágios, e também não identificaram influência do sexo no aparecimento da doença.

$\mathrm{Na}$ análise morfométrica aplicada, onde os resultados apresentados se referem ao tamanho (comprimento, altura e largura), peso e volume dos rins, e que foram avaliados considerando a idade, o sexo e a simetria bilateral, não foram verificadas diferenças quanto à simetria bilateral, uma vez que, os rins direito e esquerdo são correspondentes idênticos em relação. Também, em todos os grupos, os rins não diferem em tamanho, peso ou volume do órgão.

Estudos envolvendo o uso da imagem como ultrassonografia, de extrema valia na determinação desses parâmetros no organismo vivo, confirmam os achados da presente pesqui\&a. Assim, Sampaio e Araújo (2002), avaliaram por essa metodologia, 35 cães adultos (17 machos e 18 fêmeas), divididos em três grupos de acordo com o peso corporal (3,1-10,0 kg, 10,1-20,0 kg e 20,1-45,0 kg) e obtiveram medidas lineares renais (comprimento, altura e largura) para calcular o volume dos rins. Relataram que as medidas lineares obtidas não apresentaram diferenças significantes entre o rim direito e o rim esquerdo, semelhante ao descrito por de Barr et al. (1990), ao avaliarem também pelo mesmo método, esses parâmetros renais em cães.

Já Santana et al. (2009), através de avaliação ecobiométrica em 22 cães adultos sem raça definida (11 machos e 11 fêmeas), encontraram diferenças para as medidas de diâmetros longitudinal, dorsoventral e transversal, e volume, entre os rins esquerdo e direito, esses resultados divergentes foram relatados também em trabalhos ultrassonográficos com primatas. Hill et al. (1999) em um estudo com 136 fêmeas de macacos rhesus (Macaca mulatta), concluiu que, apesar da diferença de idade e peso dos animais, os rins exibiram tamanho similar.

Em gatos quando ocorrem diferenças mormométricas macroscópicas entre os rins, as mesmas relacionam-se a algum fator patológico, como doença renal, atrofia ou má formação do órgão (Ellenport 1986, Kealy \& MCallister 2005, Polzin 2011).

Na presente pesquisa, quando as mensurações dos rins foram comparadas entre machos e fêmeas, os resultados mostraram que os rins dos machos foram maiores para os parâmetros, comprimento, altura, peso e volume dos rins, à exceção da largura, que não apresentou diferença entre os sexos. Tais resultados nos felinos dos grupos aqui avaliados, podem estar relacionados às dimensões corpóreas do animal, uma vez que, os felinos machos apresentaram características biométricas corpóreas maiores de que as fêmeas.

Corroboram esses achados os estudos em cães de Santana et al. (2009) que encontraram uma correlação direta entre ecobiometria renal com medidas de conformação corporal. Foram comparadas a distância atlanto-coccígea (DAC) e a altura (H) de cães adultos saudáveis, com as medidas lineares e o volume dos rins. A DAC variou de $54-78 \mathrm{~cm}$ para machos e $37-71 \mathrm{~cm}$ para fêmeas e a altura variou entre $34-64 \mathrm{~cm}$ para os machos e $24-57 \mathrm{~cm}$ para as fêmeas, ou seja, cães machos apresentaram maior conformação corpórea do que as fêmeas e com isso maior volume renal, já que de acordo com o trabalho, a relação entre a DAC e a $\mathrm{H}$ com as medidas ecobiométricas renais foram significativas, demonstrando que o tamanho do rim é influenciado pela conformação corporal do cão. Esses dados também estão 
de acordo com Barr et al. (1990) que relataram haver uma correlação estatística significante entre o comprimento e o volume dos rins com o peso corpóreo.

As diferenças morfométricas entre machos e fêmeas relatadas por estes autores e encontradas neste estudo, não parecem ter uma relação direta com alterações funcionais. Mostrando que nem sempre as alterações morfológicas entre os sexos denotam alterações funcionais ou que um determinado órgão funcionaria melhor nos machos do que nas fêmeas simplesmente porque são maiores.

De fato, pois a IRIS (2009), em seu projeto piloto de avaliação de casos clínicos doentes renais, avaliou 921 animais, dentre cães e gatos, dos quais $85,7 \%(\mathrm{~N}=789)$ felídeos, e foi mostrado que a população de gatos machos foram igualmente acometidas por desordens renais à população de gatas, $51,3 \%$ e $48,7 \%$ respectivamente. Tal associação não relatou nenhuma diferença funcional entre os sexos e, portanto, a predisposição e o desenvolvimento de doenças renais não devem ser atreladas a esse fator, apesar dos relatos dos autores citados e dos resultados aqui apresentados mostrarem diferenças morfológicas entre os sexos.

Elliott \& Barber (1998) estudaram 80 casos de felinos com doença renal crônica onde foi traçado o perfil do paciente com falência renal. Fatores como sexo, idade, raça, parâmetros bioquímicos e séricos, achados clínicos dos animais, foram avaliados. Os animais foram divididos em 3 grupos de acordo com o estágio da doença: compensado, urêmico e estágio final. Em nenhum dos 3 grupos houve diferenças entre machos e fêmeas, mostrando que a doença além de afetar igualmente os sexos, não difere em relação a sua evolução entre machos e fêmeas.

Concluindo, a diferença acerca da morfometria renal entre machos e fêmeas, pode não estar diretamente relacionada somente com o peso corpóreo, mas com toda a conformação corpórea que difere entre os sexos, tais como, maior altura e comprimento do animal. 0 maior peso corporal apenas, pode não indicar necessariamente aumento na morfometria renal; assim, é necessário que se considere o escore corpóreo do animal, a ou relação com a obesidade.

Aliás, a relação entre a obesidade e a lesão renal é assunto que tem sido pouco abordado, e ainda não se encontra bem descrita. Entretanto, tem sido relatada a existência de uma relação entre alterações anatômicas associadas a uma a perda progressiva da função renal com a obesidade ou ingestão de dietas hiperlipídicas (Guyton \& Hall 2006, Paula 2006).

Em estudos com ratos, Mateus \& Fiorino (2012) notaram que uma dieta hiperlipídica (30\%) administrada durante 8 semanas a partir do desmame, causou alterações na anatomia renal. A retração significativa na área do Tufo Glomerular, e a diminuição no peso renal observadas, foram indicativos do desenvolvimento de uma patologia renal.

Para Crepaldi et al. (2008), o mau funcionamento dos rins muitas vezes está ligado a fatores genéticos; má alimentação e à pouca ingestão de líquidos. A insuficiência renal pode afetar animais de todas as raças, sexo e idade; entretanto, em animais idosos sua frequência é maior.

Com relação a idade, as comparações morfométricas aqui exibidas mostraram haver diferenças significativas em todos os parâmetros avaliados (comprimento, altura, largura, peso e volume dos rins), onde os animais do grupo 1 (jovem), apresentaram um rim menor que os animais dos grupos 2 (adulto) e 3 (senil), mas que quando se comparou indivíduos adultos com senis, essa diferença não foi significante mostrando que a partir de uma certa idade existe uma estabilidade no crescimento dos rins e em animais saudáveis a atrofia do órgão não está presente, já que animais adultos e senis não apresentaram estatisticamente diferenças em relação ao tamanho do órgão.

Como relatado por diferentes autores, as doenças renais em felinos acometem principalmente animais com idade a partir dos 7 anos. As lesões em felinos senis promovem alterações morfométricas que podem e devem ser exploradas pelo clínico para ajudar no diagnóstico da doença (Felkai et al. 1992, Nyland \& Matoon 1995, Finco et al. 1998, Espada et al. 2006, Crepaldi et al. 2008).

Estas diferenças nos parâmetros morfométricos macroscópicos entre jovens e adultos e senis, mostram um crescimento do rim em relação a idade, desde a fase juvenil contemplada neste estudo, dos 3 aos 9 meses até a fase adulta, dos 3 aos 5 anos. Este aumento dos rins nesta fase da vida do animal pode estar relacionado ao desenvolvimento corpóreo, assim como ocorre em outros tecidos do corpo, como tecido ósseo, os resultados mostraram que os rins também cresce da fase juvenil para a fase adulta.

A estabilidade morfométrica mostrada entre os animais adultos (grupo 2) e os animais com mais de 10 anos (grupo 3), revelou que não há crescimento ou aumento do rim com a idade, pois o tamanho, peso e volume não apresentaram diferenças significantes. Essa estabilidade mostra que as alterações morfológicas e morfométricas dos rins em animais com mais de 7 anos, estão relacionadas à processos patológicos e não faz parte do processo de envelhecimento.

Os relatos sobre alterações do tamanho relacionam alterações morfométricas com doença renal, alguns processos podem estar envolvidos na diminuição, como na nefrite intersticial crônica e em processos que envolvem fibrose renal, ou no aumento, como na pielonefrite aguda, na doença policística, na hidronefose, amiloidose e nefrite glomerular, dos parâmetros morfométricos do órgão (Barr et al. 1990, Sampaio \& Araújo 2002, Kealy \& MCallister 2005).

Avaliações da biometria renal em saguis, concluíram que animais que apresentavam algum tipo de alteração da sanidade, como hematúria, proteinúria e hiperglicemia, apresentaram um maior volume renal do que animais sadios, tanto na mensuração anatômica quanto na ultrassonográfica (Del Valle 2008, Agopian 2010).

Com relação as mensurações mesoscópicas, onde foram contempladas as medidas de altura do córtex, altura da camada medular do rim e a inter-relação córtex/medula, os parâmetros não apresentaram diferenças entre os rins direito e esquerdo. A manutenção da simetria bilateral e a estabilidade do órgão quando comparados os lados, reforçam a hipótese de que os rins nesta espécie não apresentam diferenças morfológicas ou morfométricas, mas apenas diferenças topográficas.

Quando considerado o sexo, os resultados morfométricos mesoscópicos não apresentaram diferenças significan- 
tes, ou seja, as mensurações de córtex, medula e sua relação não apresentaram diferenças entre machos e fêmeas.

Esta paridade entre os sexos, mostra que apesar de diferenças morfométricas na macroscopia dos rins, machos e fêmeas não apresentam diferenças morfométricas na distribuição das camadas do parênquima renal. Portanto a hipótese de que a biometria corpórea influencia na morfometria macroscópica dos rins também não se aplica às dimensões das camadas cortical e medular do órgão.

A relação morfométrica do parênquima renal, é muito utilizada em exames ultrassonográficos para detectar alterações do tecido em possíveis comprometimentos da fisiologia renal. As regiões do parênquima apresentam características acústicas diferentes, que permitem a identificação destas regiões. Assim, no corte transversal, é possível identificar a região cortical, a região medular e a pelve renal. A região cortical aparece mais ecogênica que a região medular em decorrência da presença de glomérulos, já a camada medular aparece hipoecogênica devido a presença dos túbulos do sistema coletor. Existe uma demarcação evidente entre as regiões cortical e medular, chamada de junção córtico-medular, onde se localizam as artérias e veias arqueadas (Carvalho 2004).

Através destas descrições, Rossi et al. (2012), obtiveram por meio da ultrassonografia, em condições fisiológicas, os valores de referência dos parâmetros morfométricos renais em fêmeas caprinas normais (Capra hircus) da raça Saanen em grupos de animais, com idade inferior a 6 meses, de 6 a 18 meses e com idade superior a 18 meses, e com isso estabeleceram um padrão para a espécie nas faixas etárias estudadas.

Tais características também foram utilizadas por Carvalho et al. (2010), que comparam os resultados dos achados ultrassonográficos com a histologia de tecidos renais. Dos 26 cães estudados, 11 apresentaram algum tipo de alteração no exame ultrassonográfico que compreenderam alterações de ecogenicidade, alterações no tamanho renal, alterações na relação entre as regiões cortical e medular, alteração de contorno e na ecotextura dos tecidos renais. $\mathrm{Na}$ análise histológica 17 cães (81\%), apresentaram alterações teciduais correspondentes a 11 patologias diferentes, processos inflamatórios difuso ou focal (50\%), mineralização das regiões cortical e medular (35\%), fibrose perivascular (23\%), nefrite intersticial (15\%), cistos múltiplos (8\%), degenerações glomerulares e tubulares, espessamento tubular, hidronefrose e pielonefrose.

As variações morfométricas do parênquima renal, podem sofrer variabilidade de acordo com a da idade e o peso dos animais. Dentre os 3 parâmetros avaliados nos estudos com caprinos, o córtex e a medula apresentaram maior variação de que sua relação e estavam sempre relacionadas a estes dois fatores peso e idade (Rossi et al. 2012).

Os resultados aqui apresentados demonstraram diferença significativa na altura do córtex e na inter-relação córtex-medula, com relação à idade, o que não ocorreu para a camada medular. Fato que pode indicar haver uma relação direta entre desenvolvimento do parênquima renal e faixa etária do animal, o mesmo padrão de resultados encontrados na macroscopia, quando se relaciona o tamanho do rim com a idade.
Esses resultados são pareados aos de Rossi et al. (2012), que mesmo trabalhando com outra espécie (caprina) encontrou uma variabilidade das camadas do parênquima renal relacionadas com a faixa etária do animal.

Relativamente à quantificação glomerular, foram detectadas diferenças no volume glomerular em relação ao sexo e a idade. Quando comparados, os machos exibiram maior volume glomerular, independente do grupo ao qual pertenciam. Já para a idade, o volume foi maior respectivamente para os animais do grupo 3, 2 e 1, caracterizando um aumento desse parâmetro em decorrência da idade.

As diferenças entre machos e fêmeas seguiram o padrão macroscópico, onde rins maiores, mais pesado e mais volumosos, também apresentaram maior volume glomerular; porém, sem alterações das relações do parênquima.

Apesar das diferenças morfométricas aqui relatas entre os machos e as fêmeas, a doença renal não segue o mesmo padrão, ou seja, a predisposição ou estabelecimento de lesões que geram perda de função não são descritas pelos autores, como diferentes entre os sexos. A IRIS (2009) estabeleceu que a ocorrência de lesão não obedeceu o dimorfismo sexual, 51,3\% dos acometidos por algum problema renal eram machos e 48,7\% eram fêmeas. Em 80 felinos com doença renal estudados por Elliott \& Barber (1998) os machos representaram 45 animais e as fêmeas 35. Os mesmos resultados já haviam sido relatados por DiBartola et al, (1987) quando em uma população de 74 felinos, não foi detectado predileção em relação ao sexo para a ocorrência de lesão renal.

Entretanto, nessas pesquisas, sempre é relatado a influência da idade como fator importante para o acometimento destes problemas. Assim, segundo a IRIS (2009), 697 dos 789 animais avaliados tinham idade entre 6 e 13 anos, Amador (2009) ainda destaca que 1/3 dos felinos senis são acometidos por algum problema renal.

As alterações morfológicas que levam a lesão e doença renal em felinos senis, também são descritas para outras espécies, como em caninos e em humanos, de acordo respectivamente com Lustoza \& Kogika (2003) e Slusarz et al. (2013).

A avaliação histológica do tecido renal permite a diferenciação e a caracterização de possíveis lesões do parênquima renal, e por permitir este estudo in vivo, a biópsia torna-se uma importante ferramenta no diagnóstico e diferenciação de lesões agudas e crônicas (Minkus et al. 1994; Morais 1995). 0 diagnóstico preciso através dessa análise está diretamente relacionado a qualidade da amostra analisada, e esta, por sua vez, relacionada com o número de glomérulos (Laufer-Amorim et al. 2002).

Em seu estudo com humanos, Kellow et al. (1959), relataram que a presença de quatro glomérulos por biópsia foi adequada para estudo histológico. Sem padrão definido na veterinária, vários autores descreveram estudos que visavam estabelecer um número mínimo de glomérulos por amostra biopsiada, variando de 5 a 30 (Laufer-Amorim et al. 2002).

Em cães e gatos biopsiados para avaliação do tecido renal, Pagès \& Trouillet (1990) relataram que um número maior de glomérulos na amostra permitiu uma identificação quantitativa das lesões glomerulares. 
A hipótese da variação do volume glomerular em animais com síndrome metabólica (SM) foi testada por Machado et al. (2012) em ratos submetidos a dietas hiperlipídicas, divididos em grupos que receberam tratamento para hipertensão arterial. Os autores não observaram alterações da histomorfometria renal.

Entretanto, alterações glomerulares foram descritas em ratos diabéticos, hipertrofia glomerular, acúmulo de matriz mesangial e colágeno tipo IV, lesões de podócitos, alterações endoteliais glomerulares e Inflamação renal foram agravadas no modelo diabético estudado (Hinamoto et al. 2014). Tais dados corroboram com os resultados de Agopian (2010), que encontrou um aumento da área glomerular em saguis com alterações em exames laboratoriais, como hiperglicemia e proteinúnria.

A avaliação qualitativa do parênquima renal, que exibiu diferença entre os grupos, onde foi detectada uma predominância de fibras colágenas do tipo I nos animais dos grupos 1 e 2, e fibras colágenas do tipo III nos animais do grupo 3, permite avaliações com casos de ocorrência de fibrose renal, que é descrita como fator relacionado ao envelhecimento dos tecidos, e que decoore de alterações metabólicas relacionadas a alterações de metaloproteinas da matriz mesangeal (Slusarz et al. 2013), e elevados níveis de endostatina (Lin et al. 2014).

Para Slusarz et al. (2013) o aumento da expressão de metaloproteínas do tipo 7 (MMPs-7) na matriz mesangeal, promoveu um aumento da expressão dos genes de colágeno Col1a2 e Col3a1 em ratos acima de 16 meses de idade, sugestivos de um novo mecanismo de alterações na deposição de colágeno em decorrência do envelhecimento do tecido, o que pode contribuir para o desenvolvimento de fibrose levando a doença renal crônica.

Em experimento utilizando-se ratos velhos Lin et al. (2014), verificaram que os níveis de endostatina estavam aumentados em 5,6 vezes, em comparação aos jovens, o que foi associado com rarefação microvascular e fibrose túbulo-intersticial progressiva. Os resultados foram consistentes com a hipótese de elevação dos níveis de endostatina estarem relacionados com alterações vasculares e indução de fibrose renal em ratos idosos.

Também a doença renal crônica é acompanhada pelo acúmulo excessivo da matriz extracelular, resultando em fibrose renal (Slusarz et al. 2013).

Em seu experimento para avaliar mudanças glomerulares em ratos tratados para a inibição crônica do sistema renina-angiotensina, Inserra et al. (2009) relataram que as principais alterações glomerulares presentes nesses animais com 18 meses de idade, foram reduzidas pela metade, e que a área glomerular mostrou aumento significativo com o envelhecimento nos animais sadios.

Os relatos sobre deposição de colágeno, fibrose renal e alterações da área e volume glomerular, podem estar relacionados à doenças renais, decorrentes de alterações metabólicas ou morfofuncionais. Outra hipótese a ser levantada é a de que o envelhecimento do organismo é acompanhado destas alterações que não estão relacionadas à um declínio funcional do órgão.

Tais hipóteses podem servir como orientação na busca de um maior e mais complexo entendimento dos processos morfofuncionais dos rins, e os resultados aqui apresentados podem contribuir nas descrições morfométricas e morfológicas decorrentes do envelhecimento deste órgão.

\section{CONCLUSÕES}

Em todas as técnicas biométricas utilizadas não foi detectado diferenças entre os rins direito e esquerdo.

As mensurações macroscópicas mostraram uma diferença entre os sexos, onde machos apresentaram um rim maior em comprimento, altura, largura, peso e volume do que as fêmeas, independente da idade.

A faixa etária está diretamente relacionada ao tamanho do rim, este cresce em todas as dimensões, fica mais pesado e com maior volume quando comparados jovens e adultos, e se mostra estável morfometricamente em relação aos adultos e senis.

0 parênquima renal não difere entre os sexos, mas o córtex e a relação córtex/medula difere com relação à idade, a altura do córtex e a relação com a medula aumentam com a idade.

0 volume ocupado pelo glomérulo renal é maior em machos do que em fêmeas independente da idade, também aumenta em relação à idade.

A análise histológica mostra que há predomínio de colágeno tipo I nos animais do grupo 1 e 2, e colágeno tipo III nos animais do grupo 3 .

\section{REFERÊNCIAS}

Agopian R.G. 2010. Estudo morfométrico de rins de primatas Callithrix jacchus em cativeiro. Dissertação de Mestrado em Anatomia dos Animais Domésticos e Silvestres, Faculdade de Medicina Veterinária e Zootecnia, Universidade de São Paulo, São Paulo, SP. 107p.

Almeida L.L., Mota D.L., Silva L.L.S. \& Almeida V.C.F. 2013. Efeito do extrato aquoso de Dioclea grandiflora Mart. ex Benth. nos rins de camundongos (Mus musculus Linnaeus, 1758). Braz. J. Biosci. 11(1):59-63.

Amador S.M.S. 2009. Doença renal crónica idiopática felina. Dissertação de Mestrado, Faculdade de Medicina Veterinária, Universidade Técnica de Lisboa, Lisboa. 123p.

Barr F.J., Holt P.E. \& Gibbs C. 1990. Ultrasonographic measurement of normal renal paramenters. J. Small Anim. Pract. 31:180-184.

Carvalho A.P.M., Salavessa C.M. \& Silveira L.S. 2010. Ultrassonografia e histopatologia renal em cães. Arq. Bras. Med. Vet. Zootec. 62(4):1015-1017.

Carvalho C.F. 2004. Sistema urinário: rins, ureteres, bexiga urinaria e uretra, p.111-146. In: Ibid. (Ed.), Ultra-sonografia em Pequenos Animais. Roca, São Paulo.

Crepaldi N.C.B., Tognoli L.B. \& Piccinini A. 2008. Estudo da insuficiência renal. Revta Cient. Eletrôn. Med. Vet. 10.

Del Valle C.M.R. 2008. Estudo morfofuncional de rins de primatas Callithrix jacchus em cativeiro. Tese de Doutorado em Anatomia dos Animais Domésticos e Silvestres, Faculdade de Medicina Veterinária e Zootecnia, Universidade de São Paulo, São Paulo, SP. 158p.

Dibartola S.P., Rutgers H.C., Zack P.M. \& Tarr M.J. 1987. Clinicopathologic findings associated with chronic renal disease in cats: 74 cases (19731984). J. Am. Vet. Med. Assoc. 190(9):1196-1202.

Dyce K.M., Sack W.O. \& Wensing C.J.G. 2004. Tratado de Anatomia Veterinária. 3ae ed. Elsevier, Rio de Janeiro. 872p.

Ellenport C.R. 1986. Aparelho urogenital do carnívoro, p.1481-1493. In: Rosenbaum C.E., Ghoshal N.G., Hillmann D. \& Getty R. (Eds), Anatomia dos Animais Domésticos. Vol.2. 5aㅡ ed. Guanabara Koogan, Rio de Janeiro. 
Elliot J. \& Barber P.J. 1998. Feline chronic renal failure: clinical findings in 80 cases diagnosed between 1992 and 1995. J. Small Anim. Pract. 39:78-85.

Espada Y., Novella S.R. \& Gopegui R.R. 2006. Renal ultrasound in dogs and cats. Vet. Res. Commun. 30(1):133-137.

Felkai C.S., Vorös K. \& Vrabely T. 1992. Ultrasonographic determination of renal volume in the dog. Vet. Radiol. Ultrasound 33:292-296.

Finco D.R., Stiles N.S., Kneller S.K., Lewis R.E. \& Barrett R.B. 1998. Radiologic estimation of kidney size of the dog. J. Am. Vet. Med. Assoc. 159:995-1002.

Gundersen H.J.G., Bagger P., Bendtsen T.F., Evans S.M., Korbo L., Marcussen N., Møller A., Nielsen K., Nyengaard J.R., Pakkenberg B., Sørensen F.B., Vesterby A. \& West. M.J. 1988. The new stereological tools: disector, fractionator, nucleator and point sampled intercepts and their use in pathological research and diagnosis. APMIS 96:857-881.

Guyton A.C. \& Hall J.E. 2006. Tratado de Fisiologia Médica. 11aㅡ ed. Elsevier, Rio de Janeiro, p.291-296.

Hill L.R., Hess K.R., Stephens L.C., Tinkey P.T. \& Price R.E. 1999.Comparison of kidney weight and volume to select anatomical parameters in the adult female rhesus monkey (Macaca mullata). J. Med. Primatol. 28(2):67-72.

Hinamoto N., Maeshima Y., Yamasaki H., Nasu T., Saito D., Watatani H., Ujike H., Tanabe K., Masuda K., Arata Y., Sugiyama H., Sato Y. \& Makino H. 2014. Exacerbation of diabetic renal alterations in mice lacking vasohibin-1. PLoS One 9(9):1-14.

Inserra F., Basso N., Ferder M., Userpater M., Stella I., Paglia N., Inserra P., Tenembaum D. \& Ferder L. 2009. Changes seen in the aging kidney and the effect of blocking the reninangiotensin system. Therapeutic Adv. Cardiovasc. Dis. 3:341.

IRIS 2009. Descriptive analysis of population characteristics of dogs and cats with suspected chronic kidney disease (CKD). International Renal Interest Society. Disponível em <iris-kidney.com> Accessed Aug. 27, 2014.

Junqueira L.C.U., Bignolas G. \& Brentani R.R. 1979. Picrosirius staining plus polarization microscopy, a specific method for collagen detection in tissue sections. Histochem. J. 11(4):447-455.

Kealy J.K. \& McAllister H. 2005. The abdomen, p.21-168. In: Kealy J.K. \& McAllister H. (Eds), Diagnostic Radiology and Ultrasonography of the Dog and Cat. 4th ed. Elsevier Saunders, St Louis, Missouri.

Kellow F., Cotsonas N.J., Chomet B. \& Zimmerman J. 1959. Evaluation of the adequacy of needle-biopsy specimens of the kidney. Archs Intern. Med. 104:353-359.

Laufer-Amorim R., Bandarra N.P., Monteiro Júnior L.A. \& De Moura V.M.B.D. 2002. Avaliação quantitativa e qualitativa da técnica de biopsia renal. Ciênc. Anim. Bras. 3(2):41-45.

Lin C.H.S., Chen J., Ziman B., Marshall S., Maizel J. \& Goligorsky M.S. 2014. Endostatin and kidney fibrosis in aging: a case for antagonistic pleiotropy? Am. J. Physiol., Heart Circulat. Physiol. 306:1692-1699.

Lustoza M.D. \& Kogika M.M. 2003. Tratamento de Insuficiência Renal Crônica em Cães e Gatos. Revta Bras. Med. Vet., Peq. Anim. Anim. Estimação 1(1):62-69.

Machado H., Pinheiro H.S., Terra M.M., Guerra M.O., Paula R.B. \& Peters V.M. 2012. Dissociação da resposta anti-hipertensiva e metabólica à losartana e espironolactona em ratos com síndrome metabólica experimental. J. Bras. Nefrol. 34(4):328-336.
Mandarim de Lacerda C.A. 1994. Manual de quantificação morfológica: morfometria, alometria, estereologia. $2^{\mathrm{a}}$ ed. EduERJ, Rio de janeiro. 102p.

Mateus R.S. \& Fiorino P. 2012. Estudo das alterações morfométricas renais associadas ao consumo de uma dieta hiperlipídica em ratos. Anais Sociedade Brasileira para o Progresso da Ciência, São Luís, Maranhão.

McNamara J.B., Diouf B., Hughson M.D., Hoy W.E. \& Bertram J.F. 2009. Associations between age, body size and nephron number with individual glomerular volumes in urban West African males. Nephrol. Dialysis Transplant. 24:1500-1506.

Minkus G., Reusch C., Horauf A., Breuer W., Darbes J., Kraft W. \& Hermanns W. 1994. Evaluation of renal biopsies in cats and dogs: histopathology in comparison with clinical data. J. Small Anim. Pract. 35(9):465-472.

Morais H.A. 1995. Conduta clínica nas doenças renais. Seminário Internacional Avançado de Clínica e Emergências das Doenças Renais em Pequenos Animais, São Paulo, SP.

Nyland T.G. \& Matoon J.S. 1995. Ultrasonography of the urinary tract and adrenal glands, p.95-124. In: Ibid. (Eds), Veterinary Diagnostic Ultrasound. W.B. Saunders, Philadelphia.

Pagès J.P. \& Trouillet J.L. 1990. Anatomopathological study of 142 cases of feline and canine nephropathies: usefulness of renal biopsies in everyday practice. Eur. J. Compan. Anim. Pract. 1(1):13-26.

Paula R.B. 2006. Obesidade, síndrome metabólica e progressão da lesão renal. J. Bras. Nefrol. 28(3):13-19.

Polzin D.J. 2011. Chronic kidney disease in small animals. Vet. Clin. Small Anim. 41:15-30.

Ross S.J., Osborne C.A., Kirk C.A., Lowry S.R., Koehler L.A. \& Polzin D.J. 2006. Clinical evaluation of dietary modification for treatment of spontaneous chronic kidney disease in cats. J. Am. Vet. Med. Assoc. 229(6):949-957.

Rossi R.S., Bombonato P.P., Piva F.M. \& Gregory L. 2012. Avaliação da morfometria renal através do método ultrassonográfico de fêmeas caprinas (Capra hircus) normais da raça Saanen. Pesq. Vet. Bras. 32(2):165-173.

Sampaio K.M.O.R. \& Araujo R.B. 2002. Ultra-sonografia de características lineares e estimativas do volume de rins de cães. Arq. Bras. Med. Vet. Zootec. 54(3):248-254.

Santana E.J.M., Beserra P.S., Brito A.B., Miranda S.A., Nikolak E. \& Domingues S.F.S. 2009. Doppler da artéria renal e a relação entre a ecobiometria dos rins com distância atlanto-coccígea e altura em Canis familiaris. Pesq. Vet. Bras. 29(10):809-815.

Schenck P.A. \& Chew D.J. 2010. Diet and chronic renal disease, p.181-194. In: Schenck P. (Ed.), Home Prepared Dog snd Cat Diets. Wiley-Blackwell, Iowa.

Slusarz A., Nichols L.A., Grunz-borgmann E.A., Chen G., Akintola A.D., Catania J.M., Burghardt R.C., Trzeciakowski J.P. \& Parrish A.R. 2013. Overexpression of MMP-7 increases collagen $1 \mathrm{~A} 2$ in the aging kidney. Physiol. Reports 1(5):1-20.

Spargos B.H. \& Haas M. 1994. The kidney, p.804-865. In: Rubin S.I. \& Faber J.L. (Eds), Pathology. 2nd ed. J.B. Lippincott, Philadelphia.

Stevens A. \& Lowe J. 1998. Doenças do sistema urinário, p.317-349. In: Ibid. (Eds), Patologia. Manole, São Paulo.

Tolosa E.M.C., Rodrigues C.J., Behmer O.A. \& Freitas-Neto A.G. 2003. Manual de Técnicas para Histologia Normal e Patológica. Manole, São Paulo, p.331. 